\title{
INTEGRATION OF INFRARED THERMOGRAPHY AND PHOTOGRAMMETRIC SURVEYING OF BUILT LANDSCAPE
}

\author{
M. Scaioni a , E. Rosina ${ }^{\text {a }}$, A. L’Erario a , L. Dìaz-Vilariño ${ }^{\text {b,c }}$ \\ ${ }^{a}$ Dept. Architecture, Built Environment and Construction Engineering, Politecnico di Milano, 20137 Milano, Italy - (marco.scaioni, \\ elisabetta.rosina)@polimi.it; andrea.lerario@mail.polimi.it \\ ${ }^{\mathrm{b}}$ Area of Cartographic Engineering, Photogrammetry and Geodesy, Applied Geotechnology Research Group, University of Vigo, \\ 36310 Vigo, Pontevedra, Spain - lucia@uvigo.es \\ c TU Delft - Faculty of Architecture, OTB, section GIS Technology, Delft, Netherlands - L.Diaz-Vilarino@tudelf.nl
}

KEY WORDS: Infrared Thermography, Structure-from-Motion, Close-range, Photogrammetry, Cultural Heritage, Data integration

\begin{abstract}
:
The thermal analysis of buildings represents a key-step for reduction of energy consumption, also in the case of Cultural Heritage. Here the complexity of the constructions and the adopted materials might require special analysis and tailored solutions. Infrared Thermography (IRT) is an important non-destructive investigation technique that may aid in the thermal analysis of buildings. The paper reports the application of IRT on a listed building, belonging to the Cultural Heritage and to a residential one, as a demonstration that IRT is a suitable and convenient tool for analysing the existing buildings. The purposes of the analysis are the assessment of the damages and energy efficiency of the building envelope. Since in many cases the complex geometry of historic constructions may involve the thermal analysis, the integration of IRT and accurate 3D models were developed during the latest years. Here authors propose a solution based on the up-to-date photogrammetric solutions for purely image-based 3D modelling, including automatic image orientation/sensor calibration using Structure-from-Motion and dense matching. Thus, an almost fully automatic pipeline for the generation of accurate 3D models showing the temperatures on a building skin in a realistic manner is described, where the only manual task is given by the measurement of a few common points for co-registration of RGB and IR photogrammetric projects.
\end{abstract}

\section{INTRODUCTION}

Generally, rehabilitation of the existing building stock is a key measure for reaching the proposed reduction in energy consumption and $\mathrm{CO}_{2}$ emissions in all countries. Due to the large amount of existing buildings, also a slight improvement of their energy efficiency may result in a large saving of energy at all the levels of the building's life cycle. In the case of historic buildings, the improvement of the energetic performances can be controversial with the conservation, because many products and techniques available on the market are not suitable with ancient construction materials (Pracchi, 2013). In addition, the most common software packages for the evaluation of energy efficiency do not include libraries and values for ancient materials and building techniques (Adhikari, 2013). Thus, they are generally inadequate for modeling historic buildings. Therefore, the phases of survey and diagnostics are never eliminable for the evaluation and design of the improvement in the case of historic buildings, although they are usually demanding in costs and time. In the last decades, Infrared Thermography (IRT- see, e.g., Vollmer \& Müllmann, 2010) has been contributing up to an impressive extent to the investigation of thermal behaviour of buildings, including the ones belonging to Cultural Heritage (CH), chiefly for the maintenance/conservation of façades and the evaluation of the building thermal performances (Grinzato, 2012; Rosina, 2013). The latter is particularly cutting edge application because of the increasing need to improve the energy efficiency of historic buildings as well. This objective has been recently recalled by international and national standards, see, e.g., CEN 16883/16 guidelines (Italian Ministry, 2015). The overlap of thermographic images, optical RGB digital photos and their localization on the building façades is, of course, a fundamental praxis for the study of thermal anomalies. On the other hand, in the case of ancient buildings (but not only), the geometry of the construction may strongly affect the thermal behaviour. For example, the walls to investigate may not be regular, since thickness, structure, materials and number of layers may be different even in the same part of the construction. Therefore, the metric location of thermal anomalies that should be further investigated (often also with destructive tests) is crucial for reducing as much as possible the size and number of samples to collect and analyse.

The outstanding improvement of surveying solutions able to gather detailed 3D models of buildings has furnished and adequate geometric support for mapping IRT data in their precise locations. Mainly, 3D modelling (Stylianidis \& Remondino, 2016) may be based on Terrestrial Laser Scanning (TLS), and Close-Photogrammetry - including drone-based Photogrammetry, but other techniques have been also investigated (e.g., range cameras, see Altuntas et al., 2016). As the state of the art comprehends some basic techniques for mapping IRT imagery on simple building models, the integration of 3D models derived from TLS/Photogrammetry to IRT data allows the generation of accurate 3D multispectral models useful for localization, visualization, and analysis of thermal anomalies in buildings (Scaioni et al., 2012).

After information extraction, these data sources should be implemented in the advanced tools for the management of building information, such as Building Information Models (BIM’s), see (Saygi \& Remondino, 2013). Regarding the modeling of 3D data and IRT, the integration into a BIM (Building Information Model) could represent the optimal solution for works management and decision-making assessment, due to their capacity to coordinate all the 3D information needed for the diagnosis of the building and the planning of the rehabilitation works. In Previtali et al. (2012), the integration of 2D outputs of thermal analysis of single façades into a GIS-desktop environment was proposed. This solution was effective for data representation and comparison within multitemporal data sets, but suffered from the absence of 3D reference and was disconnected from the real 3D structure of the building under investigation. Since TLS, infrared thermography 
and SfM photogrammetry are techniques that acquire information of the object as-it-is, the resulting BIM includes information on the real condition of the building in the moment of inspection, consequently helping to a more efficient planning of the rehabilitation works, enabling the repair of the most severe faults. Expected results are the improvements of diagnostics and survey as well as shortening the time from data analysis to preliminary restoration design and improvement of the performances. Moreover, the availability of results in the preliminary phase allow to address the executive projects since the beginning, avoiding expensive changes of orientation in the further steps of the study/projects.

In the following, the authors discuss some attempts to increase the automation of the process of integrating surveyed 3D models and IRT, either in the phase of data acquisition and data modeling. In either the former problem the integration of geometric data, visible and IR texture is not yet a fully automated process both using TLS (Costanzo et al., 2015) or digital photogrammetry (Previtali et al., 2013). Although at present the acquisition techniques allow to obtain dense point clouds in a relatively easy way, mapping thermal data very often require an additional modelling step, which may be time consuming (Previtali et al., 2014). On the other hand, the consolidation of Structure-from-Motion (SfM) photogrammetry (Barazzetti et al., 2011) offers today the opportunity to obtain point clouds of building façades in almost automatic way, whilst the cost for hardware and software equipment also may remain quite small with respect to TLS pipeline.

In previous papers, the authors have investigated the approach for integrating TLS 3D models and IRT collected using a groundbased thermal cameras. In these solutions, reviewed in Section 2 together with the current state-of-the-art of this IRT, the geometric model to support the texture from IR images is given by TLS data. This technology is fully capable of providing accurate 3D models, but with still high cost of the equipment and non-complete versatility of usage, in particular because of the instrument size and weight. On the other hand, even though the standard formats for IR thermal cameras are slowly increasing (now we may consider the $640 \times 480$ pixels as the basic format size), in order to accomplish a detailed survey of a large objects several tens or hundreds of thermal images may be necessary. The registration of individual images to the 3D model using space resection (Luhmann et al., 2014) would require too much effort, on one side for the necessity of manually measuring a terrific number of control points, on the other because the identification of such points on a purely geometric model (without photorealistic texture) may be a complex task. The solution proposed in Alba et al. (2011) and Previtali at al. (2013) instantiated a new approach exploiting RGB images to help co-registration between 3D model and IR images (see Sect. 2). On the other hand, in the latest here the improvement of dense matching techniques (Remondino et al., 2014) and their implementation of powerful SW packages to accomplish the partial or complete SfM-Phot pipeline (Hartmann et al., 2016).

The rest of this paper is organized as follows. Section 2 reviews recent advances in the reconstruction of $3 \mathrm{D}$ thermal models. Section 3 deals with the qualitative analysis of thermograms while Section 4 describes the proposed methodology exploiting up-to-date techniques for image-based 3D modelling (i.e., SfMphotogrammetry and dense matching) along with the application to a real case study. Section 5 regards the discussion of the results and the conclusion.

\section{STATE-OF-THE-ART AND RESEARCH DIRECTION}

Despite the documented benefits of using IRT to $\mathrm{CH}$ conservation (Grinzato, 2012), the current practice still bases on the direct application of 2D thermal images (Cho et al, 2015).
This practice has several inefficiencies, including the absence of metrics and low spatial resolution and narrow field-of-view (FoV) of thermal cameras, which makes necessary the collection of a large amount of images to depict completely a building façade. These weaknesses become more relevant in case of $\mathrm{CH}$ façades in which the complexity of their geometry can affect to the quality of the thermal survey. Over the last few years, high efforts have been made towards the automatic generation of 3D thermal models in which the thermal and the metric information of a facility are integrated. Several methods were developed, mainly belonging to two groups whether the metric information is obtained from an external data source such as TLS or from photogrammetric procedures. On one hand, mapping thermal images on a three-dimensional model created with laser scanner has been widely used to create 3D thermal models. Basically, the process consists on applying a texture directly to the point cloud or to a mesh-model created from it. For this purpose, the intrinsic calibration of the IR camera and the co-registration of both thermal camera and laser scanner are a mandatory requirement. However, the reduced spatial resolution and the narrow FoV of thermal cameras make these processes not trivial (Alba et al., 2011). Several approaches aiming to automate the mapping of thermal images onto point clouds have been recently presented. The use of an auxiliary RGB camera helps in the computation of the thermal images orientation. Alba et al (2011) developed a 'bicamera' system coupling a thermal IR camera to a RGB camera. RGB images are processed through a photogrammetric bundle adjustment, and as the relative orientation between RGB and IR cameras is known, thermal images are oriented. Previtali et al (2013) compute an integrated bundle adjustment of RGB and IR imagery, after the preliminary independent calibration of both sensors. After that, texture is applied to a 3D mesh created from the point cloud. González-Aguilera et al (2012) implemented a method based on the extraction of common features in IR images and in ranging images from the point clouds. An image-matching process is followed to register both sensors and texturize the 3D point cloud. Lagüela et al (2013) implemented the linear feature detection to register thermal images with RGB images. Once thermal images are oriented, they are used to texture 3D mesh models created from the point clouds. Bormann et al (2013) developed a mobile inspection system including a laser scanner and a low-resolution thermal camera. The calibration between the camera and the laser scanner is used to automatically map colour from images onto the point cloud. Sequential scans are registered by using a $6 \mathrm{D}$ simultaneous localization and mapping technique (SLAM). Golparvar-Fard and Ham (2014) proposed a method to extrapolate thermal information to improve model completeness. Textured and non-textured points are structured into a k-d tree (Samet, 2006) and the nearest neighbour search is used to assign thermal values to the non-textured points.

On the other hand, important progresses have been recently made in the automation of image-based 3D reconstruction procedures, whereby 3D models are generated from an unordered collection of photographs. Most of progresses in this field come from the computer vision community, and specifically from the advances in feature detection and matching techniques. SfMphotogrammetry (Snavely at al., 2008; Westoby et al., 2012) is already a consolidated method to obtain point clouds from RGB images with a relatively high quality and in a quick way. With regard to the application of SfM to thermal images, few works have been presented in the last years. Ham and Golparvar-Fard (2013) investigated the performances of feature detection and matching algorithms including SIFT, ASIFT and SURF for large sets of unordered thermal images. Results exhibit very low matched keypoints between pairs of thermal images in comparison with digital images from the same scene, making them insufficient for registration. This behaviour is mainly 
explained by the gradual variations of surface temperatures, usually represented as colour gradients, causing the absence of abrupt features and hampering feature detection and correspondence. Another approach, developed by Ham and Golparvar-Fard (2013), is based on the simultaneously caption of thermal and digital images. For each pair, the relative pose is calculated and a SfM procedure is applied to digital images to estimate their pose in $3 \mathrm{D}$ and in this way, thermal image poses. Finally, a Multi-View Stereo algorithm is applied to produce dense 3D thermal point clouds. The generation of 3D thermal models from photogrammetric procedures reduces the cost of the survey since no TLS sensor is needed. However, the main disadvantage of applying these techniques to thermal images is the low feature detection rate between pairs of images. When using RGB images to estimate the pose and calibrate thermal images, results improve significantly although the resulting 3D models can be partially incomplete and noisy.

\section{EXPERIMENTAL SECTION}

The significant steps forwards of the techniques for detecting, mapping, improving the localization of thermal anomalies on the building envelopes allowed to enlarge their application to the diffused stock of existing buildings, listed and not listed, that require the improvement of performances for energy sparing. In the present paper, IRT application mainly focuses to distinguish the thermal anomalies of building façades at different environmental conditions, for detecting both damages and thermal loss. The inspection of building envelopes by IRT bases on the effects of heat flow across the structure. The surface temperature is a function of heat flow crossing the wall and local boundary conditions. This parameter may give information regarding the interior layers of the structure. The heat is transferred more quickly throughout the most cohesive materials or/and materials with greater thermal diffusivity. Any detachment of the finishing layer strongly reduces the heat transfer and adds its signal to that given by the structure. In addition, also moisture affects the heat transfer across the structure, and the localization of damp parts is crucial both for the evaluation of transmittance and the early detection of a risk for the conservation of materials. The presented study cases consist on a protected building, a church dating back to 60's, and a residential building dating back to same time. Both constructions are located in Milano, Italy. The choice of these case studies depends on their poor insulation, typical of the building techniques of that time in Italy: they are representative of a large amount of existing buildings that need to go under refurbishment to improve their energy efficiency, according to the present regulation. The authors used an IR Thermocamera Flir P64. The IRT equipment has a FPA microbolometer VOX

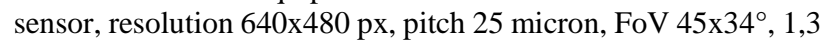
mrad, objective $19 \mathrm{~mm}$.

\subsection{Case Study 1: The St. Pio X Church}

The St. Pio X church (see Fig. 1) has a reinforced concrete structure. The finishing of the inspected façade is clinker, in a poor condition for lacking maintenance in the last decades. The church has a heating plant, radiant panels in the floor that was in function during the IRT recaptures. The following images show the thermograms of the church façade shot at different environmental condition, as an example of the information that IRT displays in real time, in a preliminary phase of the image processing.
Two recaptures included 18 images shot frontally and with an angle of $15-20^{\circ}$ from the central axis of the façade.

During the solar irradiation, the damages of the clinker finishing determine the temperature pattern that is visible on Figure 2.

The warmer areas (red squares, continuous line) indicate the presence of tiles detachments: the heat flux due to the solar irradiation slowly flows across the structure because of the air gap underneath the tiles, causing higher temperatures of the surface if compared with the surrounding zoned where the finishing is sound and the heat flows more quickly across the structure. The black areas (in the red circles, spotted line) indicate the missing tiles: the lighter color of the substrate (and its thermal properties different from the tile ones) causes the different heat absorption, and different surface temperature. The colder strips (red rectangles, dotted line) are due to the presences of the rebars in the reinforced concrete: the metal is more conductive than the cement mortar and tiles; therefore, the heat flux penetrates in the metal faster than in the concrete.

This first step of the analysis shows very apparent defects in real time; nevertheless, the lack of geometric reference allows to have only a qualitative estimation of the extension of the damages, without a reliable metric evaluation of the size. A rectification in the post-processing phase it will be very helpful to design a proper maintenance plan and its economic evaluation.

\subsection{Case Study 2: Residential building in Piazza Leonardo da Vinci, Milano}

The second application deals with the evaluation of the energy efficiency of buildings. The object of this second analysis was a residential building built up in the 60's in Milano, very close to Case Study 1 (see Fig. 4). This construction is a typical example of the so-called diffuse construction that today pervades the European cities because of the impressive urbanization growth occurred in the decades after World War II.

The authors shot two recaptures: a preliminary scanning of 30 images that served to localize the thermal loss (at the beginning of February), and a second scanning of 115 IR images, with a superimposing ratio of $80 \%$ for generating the 3D model (later, at the end of March). In this second recapture, the images were acquired with a regular pace of $1.5 \mathrm{~m}$, at a distance of $15 \mathrm{~m}$ from the façade.

In a cloudy, winter day, at dawn, with a low diffused irradiation, the distribution of the surface temperature is mainly due the heat flowing from inside towards outside. The temperature distribution on the façade is due to the materials of the envelope: the higher temperatures are located where the structural beam above the entrance, the pillars and the vault are joint to the façade. The poor insulation of the structures causes the thermal loss that determines the higher temperature of the surface. Also in this application, the modeling phase of the surface will be very helpful to locate exactly the thermal anomalies and to share diagnostics data with all the designers and experts involved in the process of improving the energy efficiency of the building. As anticipated in Section 1, the use of BIM resulted a great improvement; nevertheless, the time and costs to realize the model prevent the application to the common stock of existing building. The automatization of the process, showed in the next paragraph, will improve the application with the result to spread the use as a commonly viable practice for the maintenance of all the existing buildings. 


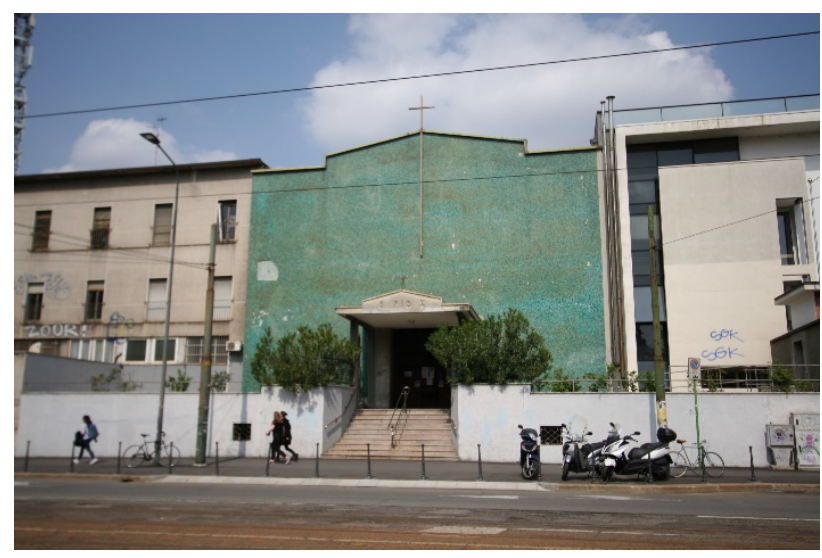

Figure 1 - The southern façade of St. Pio X Church (Case Study 1), facing Piazza Leonardo da Vinci square.

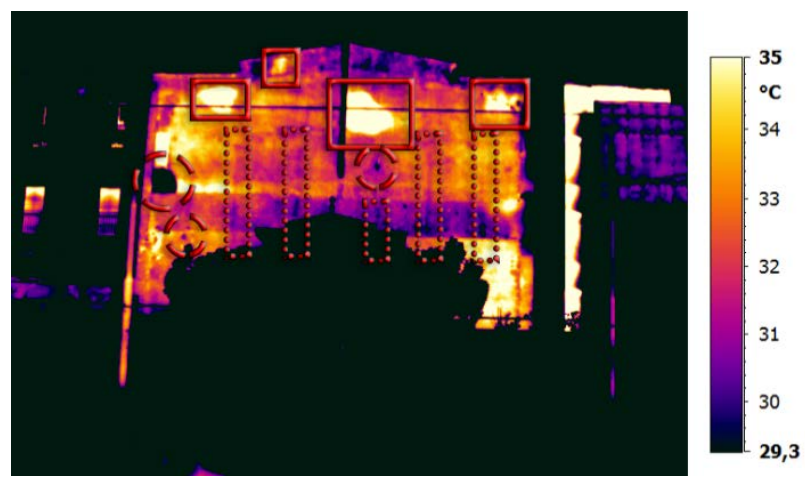

Figure 2 - Thermogram of the southern façade of Case Study 1 during the solar irradiation, air temperature $12^{\circ} \mathrm{C}$; emissivity 0.92 . The black part at the bottom is due to the trees and entrance porch that were not an objective of the present investigation; therefore, the optimized range of temperatures excluded that colder part of the image.

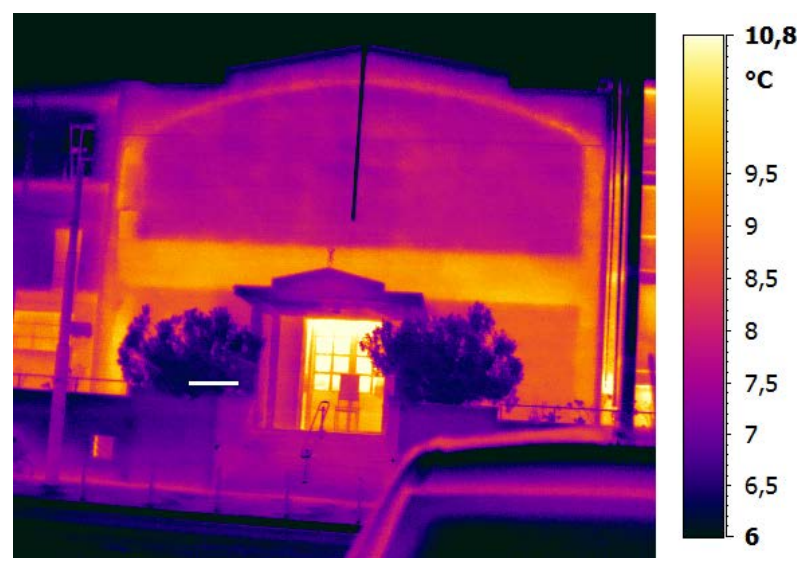

Figure 3 - Thermogram of the southern façade of Case Study 1 , captured at dawn before the solar irradiation. The indoor air temperature was $18 \mathrm{C}$, outdoor $7 \mathrm{C}$, emissivity 0.92 .

Two different steps have been accomplished for the analysis of Case Study 2. In the first one IR images have been analysed independently from the integration to 3D model. Results are reported in the sequel of this subsection. In the following Section 4 , a methodology to operate a fast mapping of IRT data on a 3D model derived from SfM-photogrammetry will be described. The multi-story building has a solid masonry structure; the inspected façade has differentiated finishing: stone slabs at the bottom and sides, plaster and clinker in the middle. The heating plant consists in radiators located below the windows locally operated, therefore some of them were not functioning at the time of the recaptures. The purpose of the preliminary analysis is the detection of thermal loss on the façade during the heating of the building at optimal environmental conditions (at dawn, in a cloudy winter day, with a difference of about $10^{\circ} \mathrm{C}$ across the building envelope).

The thermogram in Figure 5 shows higher temperatures in some spots of the façade due to:

1. The location of heaters, where the façade is plastered (red circles, continuous line);

2. The ineffective insulation of the slabs (red rectangles, dotted line), both where the finishing is plaster (the upper floor) and stone slabs (the ground and first levels); and

3. The ineffective insulation of the vertical heating pipes (red vertical rectangles).

The heat flux across the masonry affects the surface distribution of temperatures where the thickness of the masonry is lower and its thermal properties facilitate the transmission of the heat from inside towards outside.

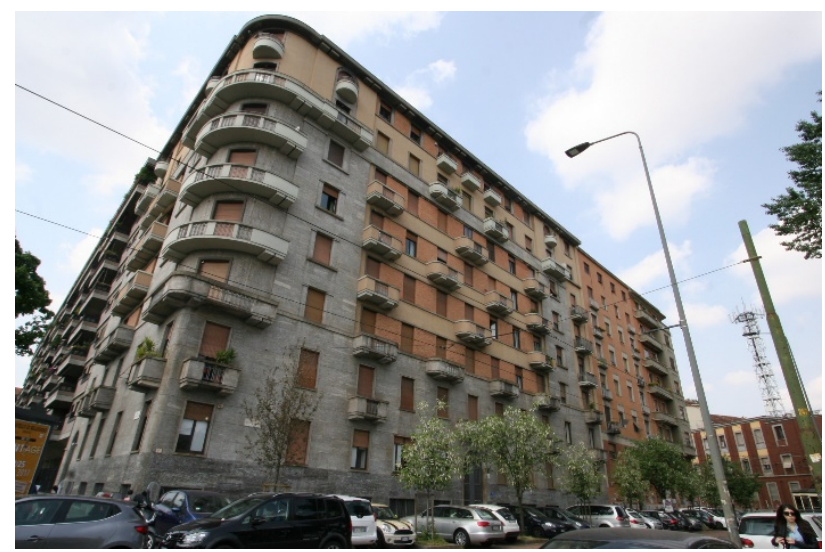

Figure 4 - Eastern façade of the residential building in Piazza Leonardo da Vinci square, Milano, Italy (Case Study 2).

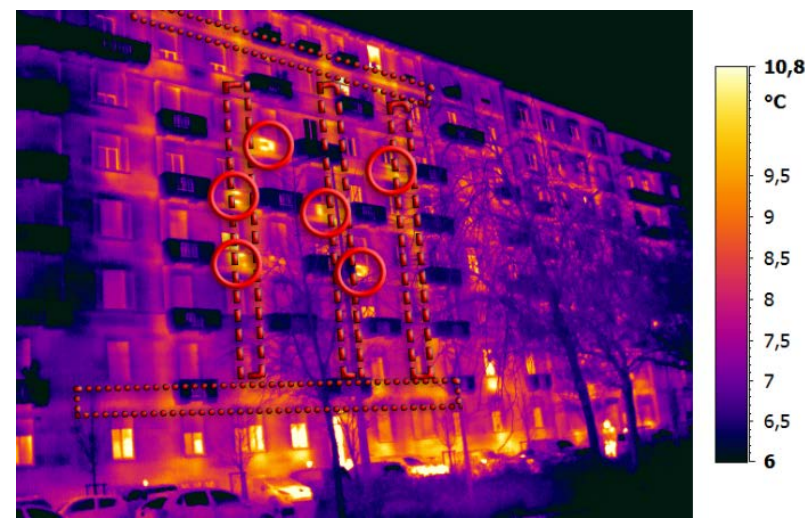

Figure 5 - Thermogram of the eastern façade of Case Study 2; outdoor air temperature $8 \mathrm{C}$, emissivity 0.92 .

IRT also provides information on the structure pattern itself. As an example, Figure 7 shows the temperature distribution that depends on the composition of the masonry (a red arrow 
highlights the area). The warmer temperatures are due to the presence of squared elements in this part of the masonry (maybe large hollow bricks) together with the higher temperature of the water in the vertical pipes, and the thinner finishing (clinker). At optimal environmental conditions, the thermal definition of the images allows locate the thermal loss (Fig. 8); nevertheless, the perspective and angle of recapture prevent any precise estimation of the extension of the thermal loss. In addition, the presence of prominent balconies prevents any analysis on the surfaces above or around them.
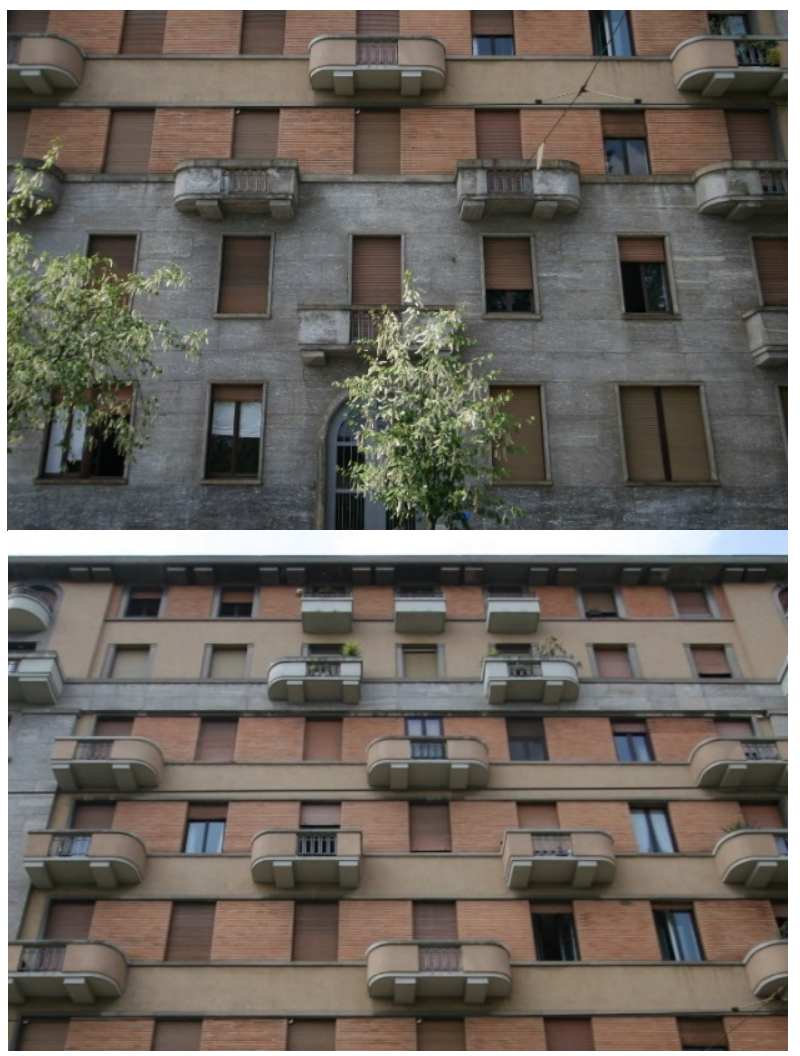

Figure 6 - Details of the lower (at top) and lower part of the Case Study 2's façade.

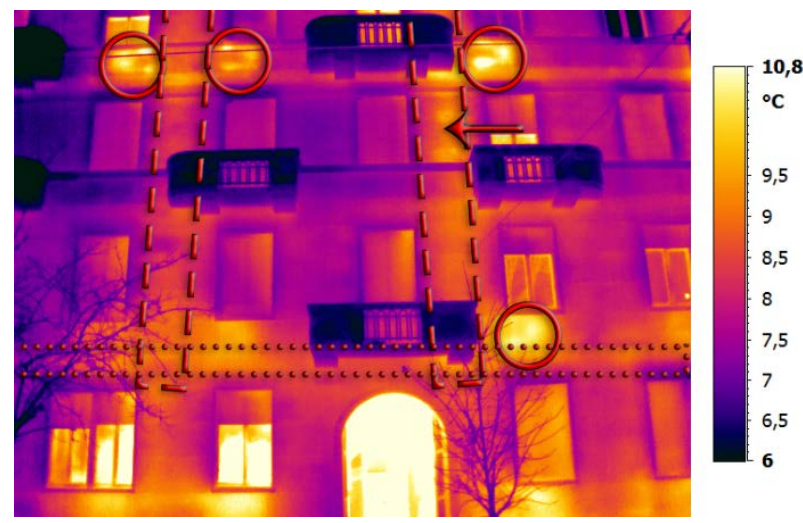

Figure 7 - Detail of the temperature distribution on the area around and above the main entrance (Case Study 2). The thermogram shows the heat loss due to the different thickness of the masonry, its finishing, and the location of heater/pipes.

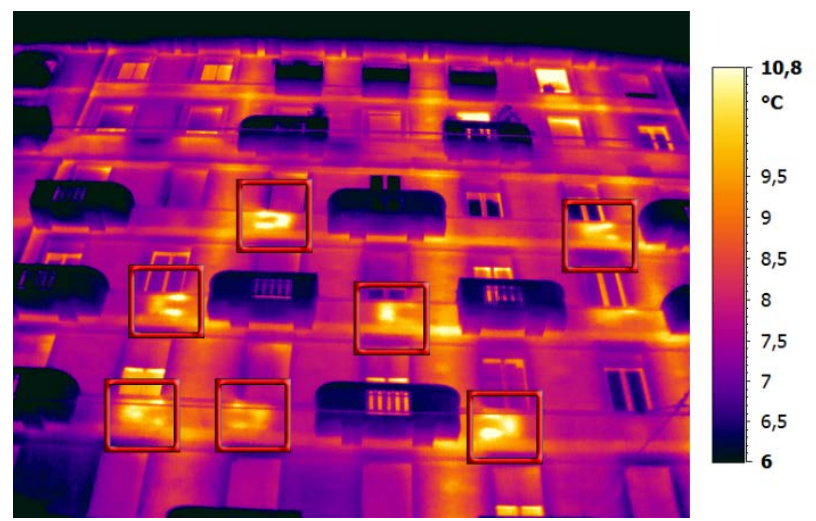

Figure 8 - Detail of the temperature distribution on the upper part of the façade (Case Study 2), above the main entrance. In the red squares, the warmer areas due to the heat flux generated by the heaters. The reduced thickness of the masonry where the heaters are located causes higher surface temperatures, especially at the connections between the heater and the hot water pipes.

\section{CREATION OF AN ACCURATE 3D MODEL TEXTURED WITH IRT DATA}

\subsection{Methodology}

The approach proposed here for mapping a 3D geometric model with thermal images has been though two fulfil the following requirements: fast and simple data acquisition, low-cost of required $\mathrm{HD} / \mathrm{SW}$, automation to speed up the processing stage, and possibility to exploit commercial HD/SW solutions. These properties are necessary to allow the use of this methodology in a large extent of real cases, as well as to make possible the application in developing countries or in situations with limited budget.

First, the technique adopted for geometric modelling is SfMphotogrammetry applied to a block of RGB images. After data acquisition, automatic orientation, sensor self-calibration and dense matching are operated using standard procedures. The model obtained in this way can be geo-referenced by using accurate Ground Control Points (GCP's) obtained from theodolite measurements, rather than using less accurate points from a digital map and a scale bar more precise scaling. Captured images should feature adequate scale for enabling the reconstruction of a model with level of detail adequate to the resolution on the object of thermal images. This aspect is particularly important especially for the correct texturing in correspondence of edges (for example, around windows). In fact, a good resolution of geometric modelling may prevent the use of editing or vectorization of 3D point cloud, which was an important step highlighted in previous studies to obtain a correct thermal texturing (Previtali et al., 2014). Indeed, from the dense point cloud that can be automatically obtained from photogrammetric processing, a 3D TIN (Triangulated Irregular Network) model is generated to be used for successive thermal texturing. Of course, the use of image-based approach also allows one to obtain the realistic texturing of $3 \mathrm{D}$ model in straightforward manner.

The same operation is repeated with a block of thermal IR images, but halted to the image orientation phase since 3D modelling will be based on the TIN surface derived from RGB images. In the literature, the possibility of using a thermal camera as photogrammetric sensor has been widely demonstrated, mainly based on preliminary calibration using GPC's (see 
Gianinetto et al., 2005). Here an approach based on selfcalibration using the same data set captured for texturing the geometric model is applied. This means that the block made up of IR images should include:

1. One or more strips of images roughly parallel to the building façade, with suitable image scale and forward overlap of approximately $80 \%$;

2. Some convergent images to strengthen the block geometry and also with the secondary purpose of texturing extruding or protruding elements (e.g., balconies); and

3. A few rolled images (approximately $90^{\circ}$ ) to help decorrelating the estimate of interior orientation ad additional parameters for correcting lens distortion; indicatively, at least one rolled image should be captured every 8 images following criteria at points 1 and 2.

The computation of image orientation including camera selfcalibration is done by using a standard SfM approach (Barazzetti et al., 2011). A free-net Bundle Adjustment (BA) may be operated in a first step, to be followed by a constrained BA where GCP's may be included to the purpose of geo-referencing and to enforce block stability. GCP's may be also derived from intersection of corresponding points measured in the RGB images. This task is main point that is still based on interactive measurements. In fact, as remarked in Section 2, automatic registration of RGB data set and IR data set is still an open problem, for which a quite general solution has not been consolidated yet.

Once the TIN surface model has been derived from optical images (of course, it may come from TLS surveying or from existing building model, if already available) and IR images have been registered into the same reference system, thermal texture can be projected on the 3D model. Other by-products as orthoimages may be also derived at this stage.

\subsection{Experimental application to Case Study 2}

The pipeline described in previous subsection has been applied for thermal mapping of the residential building in Case Study 2.

\subsubsection{Reconstruction of the geometric model}

A photogrammetric block consisting of 182 RGB images has been gathered using a Nikon D80 camera (CCD sensor, size $24 \times 16 \mathrm{~mm}$, 3872x2592 pixels, $6.2 \mu \mathrm{m}$ pixel size) equipped with a $18 \mathrm{~mm}$ lens. Images have been shot from the ground floor in the square in front of the building to obtain a pixel footprint ranging from $2 \times 2 \mathrm{~mm}$ up to $5 \times 5 \mathrm{~mm}$, depending on the building floor. In Figure 9 the camera poses are depicted after image orientation. The RGB block has been designed to have large overlaps to increase inner reliability and to cover all parts of the façade, with the exceptions of those shadowed by balconies. These would have required elevated acquisition standpoints or drones to be accomplished. Processing of RGB images has been operated using Agisoft Photoscan (ver. 1.2.4), implementing a typical SfM procedure. A number of tie points (TP's) per image ranging from 1918-12950 (average \#4958) has been successfully matched, to be used in BA for estimating camera poses and camera calibration parameters. Root Mean Square (RMS) of residual of TP's in image space has resulted 0.14 pixels. Approximate geo-referencing of the RGB block has been done be using some information derived from the spatial database of Milan Municipality, integrated by the measurement of a few baselines for scaling. Fifteen points have been measured on the left part of the façade to be used as GCP's for co-registration of the IR block. Eventually, a dense point cloud has been generated from oriented images using dense matching functionality, and then a TIN model has been computed (see Fig. 9). No further specific tasks for independent quality assessment of the geometry of this block has been accomplished (Nocerino \& Remondino, 2016), but the high-redundancy and the analysis of quality figures provided by Photoscan software are retained sufficient to the purpose of this study.

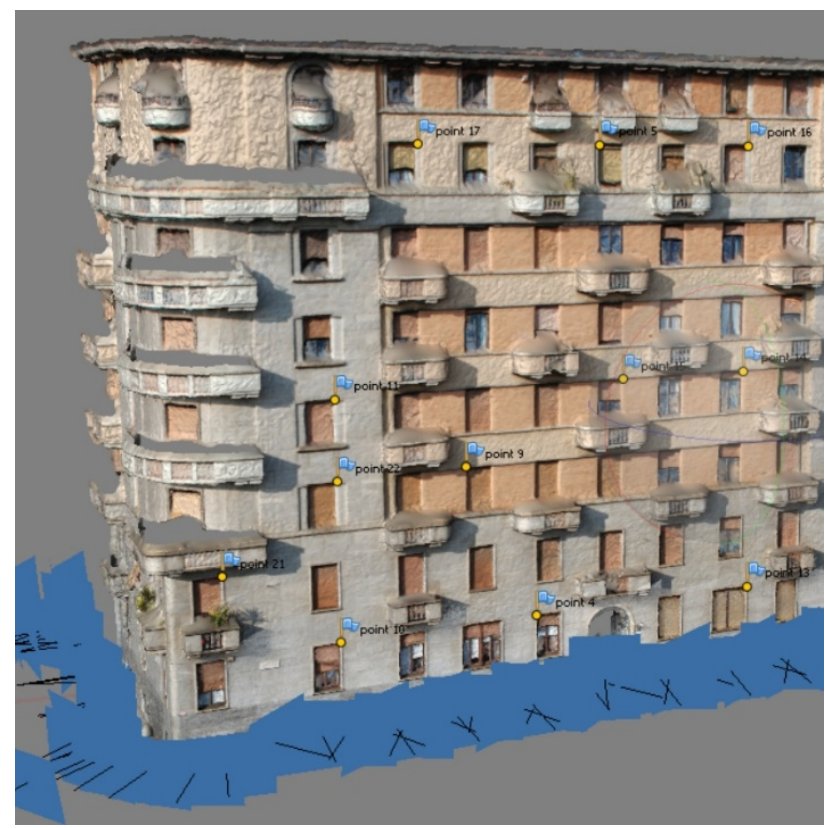

Figure 9 - Print-screen displaying the result of photogrammetric processing of RGB images in Case Study 2: the TIN model of the building façade textured with RGB photos, the location of extracted GCP's for co-registration of IR images, and estimated camera poses.

\subsubsection{Data acquisition and orientation of IR images}

During data acquisition for capturing IR images described in Subsection 3.2, attention has been also paid to collect images following a block geometry suitable for photogrammetric reconstruction (see criteria in Subsect. 3.1). Only the left part of the façade has been imaged. This choice has not been merely motivated by limiting the sample to process, but because data acquisition should not span over too much time to keep similar environmental conditions during all shots. Images have been organized in two strips covering the lower and upper part of the façade in Case Study 2. A pixel footprint ranging from $4 \times 4 \mathrm{~mm}$ up to $8 \times 8 \mathrm{~mm}$ has been obtained, depending on the building floor. In Figure 10 the IR camera poses are depicted after image orientation.

Processing of IR images has been operated using Agisoft Photoscan (ver. 1.2.4), following a similar procedure as done for RGB image block, but limited to estimate image orientation. The number of TP's per IR image has been in the range between 51 and 951 (average \#545), with average residuals in image space of 0.32 pixels. During BA, the estimate of IR camera calibration parameters has been also included. In order to limit the number of parameters to use, the following coefficients of the Brown's models (Luhmann et al., 2016): principal distance (c), and principal point $\left(x_{p p}, y_{p p}\right)$, symmetric radial distortions $\left(K_{1}, K_{2}\right)$, , symmetric radial distortions $\left(P_{1}, P_{2}\right)$. 
Among fifteen GCP's available, 8 have been measured on the IR images for co-registration to the RGB block. Average residuals have resulted as $2.5 \mathrm{~cm}$ in object space and 0.32 pixel in image space. These results have been positively evaluated for using these IR images to texture the TIN model. Eventually texturing has been accomplished to obtain the IR-textured model in Figure 10. The model has been retained to fulfil preliminary requirement. Of course, some holes reamin in Figure 10 due to: shadowing effects because of balconies and the presence of trees very close to the façade (see Fig.'s 4 and 6), incompleteness of the TIN model.

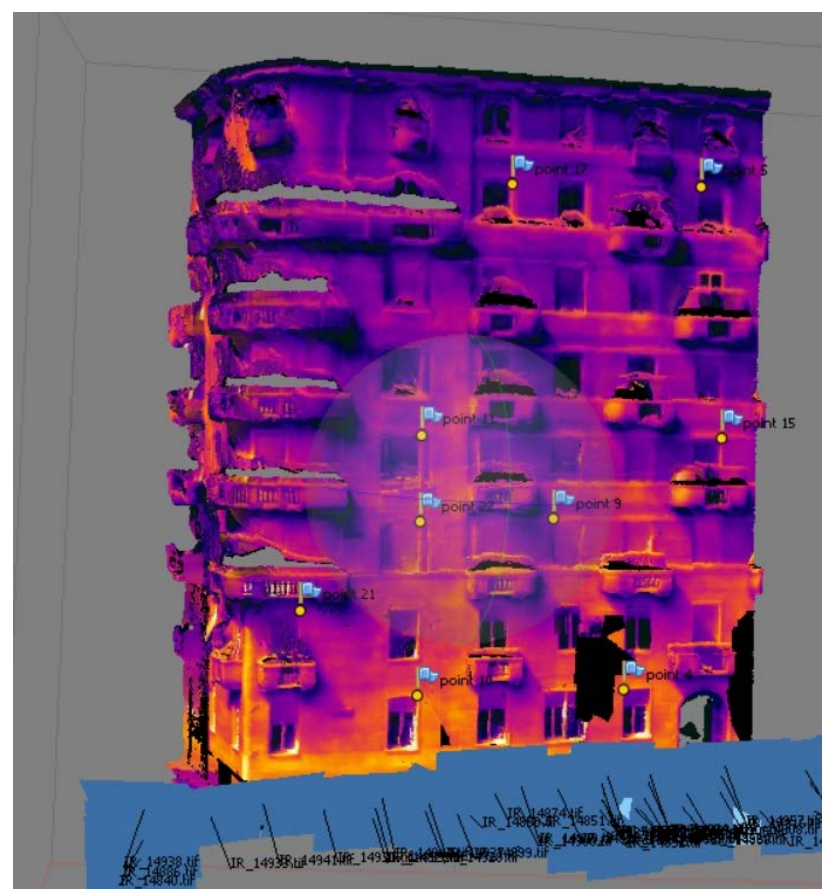

Figure 10 - Print-screen displaying the result of photogrammetric processing of IR images in Case Study 2: the TIN model of the building façade textured with IR images, the location of extracted GCP's for co-registration to RGB data set, and estimated IR camera poses.

\section{FINAL DISCUSSION AND FUTURE WORK}

The paper reports the discussion on the application of Infrared Thermography (IRT) for the analysis of thermal anomalies of Cultural Heritage and existing buildings. In particular, the analysis of a couple of case studies in Milan, Italy helped to focus and discuss two different aspects, regarding the assessment of the damage and the energy efficiency of the building envelope. In the second part of the paper, the integration of IRT surveying and Photogrammetry has been dealt with to provide detailed 3D thermal models where the possible geometric complexity of a building may be also accounted for in the analysis. After reviewing the state-of-the-art in Section 2, the focus was given to demonstrate the possibility of applying a fully image-based procedure for 3D modelling and IR texturing. This approach has on one side the advantage w.r.t. the use of laser scanning technology of a major budgetary sustainability. On the other side, it allows to exploit the high potential of Structure-from-Motion for image orientation and camera calibration, as well as dense matching procedure for 3D surface reconstruction. The application of these techniques resulted in a quite fully automated processing pipeline, where the only manual task is the measurement of common points to register the digital surface model derived from dense matching and the block of IR images to be used for texturing thermal data. Of course, efforts to overcome this step and to obtain a fully complete automatic pipeline require the attention of researchers, considering that a few positive experiences were already reported in the literature (see Sect. 2). Another important aspect to investigate is the optimization of IR data acquisition, since the images should be quickly captured to avoid significant changes in the environmental conditions that would bias the output of the thermal analysis. As an alternative, the recaptures should be done at steady condition, without any source of heating hitting the surface and constant heating inside the building. In this second case, the condition are optimal for the analysis of the energy efficiency of the building envelope and to inspect the structure of the building, as well as the location of air7water leakages and rising damp; on the contrary, steady condition could prevent good results of the analysis of the surface defects, that requires a direct thermal stimulation.

\section{Acknowledgements}

Acknowledgements go to Milan Municipality for providing the spatial database. The authors would also thank Anna Privitera, Eliana Tonelli, Riccardo Valente for the help with the Case Study 2 photogrammetric reconstruction, Lucia Bersi and Sidorela Hasani for their help with the recapture and processing of IR thermograms. The fourth author would like to give thanks to the Xunta de Galicia for the financial support given through human resources grant (ED481B 2016/079-0).

\section{REFERENCES}

Adhikari, R., Pracchi, V., Lucchi, E., 2013. Energy modelling of Historic Buildings: Applicability, problems and compared results'. In: Proceedings of EWCHP 2013, 3rd European Workshop on Cultural Heritage Preservation, Bozen, Italy, pp. 219-225.

Alba, M.I., Barazzetti, L., Scaioni, M., Rosina, E., Previtali, M., 2011. Mapping Infrared Data on Terrestrial Laser Scanning 3D Models of Buildings. Remote Sensing, 3(9), pp. 1847-1870.

Borrmann, D., Elseberg, J. , Nüchter, A., 2013. Thermal 3D mapping of building façades, Intelligent Autonomous Systems, Springer, Berlin Heidelberg, 12/2013, pp. 173-182.

Italian Ministry, 2015. CEN 16883: Conservation of Cultural Heritage - Guidelines for improving energy performance of historic buildings.

Cho, Y.K., Ham, Y., Golpavar-Fard, M., 2015. 3D as-is building energy modeling and diagnosis: A review of the state-of-the-art, Advanced Engineering Informatics, 29, pp. 184-195.

Costanzo, A., Minasi, M., Casula, G., Musacchio, M, Buongiorno, M.F., 2015. Combined use of terrestrial laser scanning and IRT thermography applied to a historic building. Sensors, 15/2015, pp. 194-217.

Golparvar-Fard, M., Ham, Y., 2014. Automated diagnostics and visualization of potential energy performance problems in existing buildings using energy performance augmented reality models, Journal of Computing in Civil Engineering, 28, pp. 1729.

González-Aguilera, D., Lagüela, S., Rodríguez-Gonzálvez, P., Hernández-López, D., 2013. Image-based thermographic modeling for assessing energy efficiency of buildings façades, Energy and Buildings, 65, pp. 29-36. 
Grinzato, E., 2012. IR Thermography Applied to the Cultural Heritage Conservation. 18th World Conference on Nondestructive Testing, 16th-20th April 2012, Durban, South Africa.

Grinzato, E., Bison, P., Girotto, M., Volinia, M., 2009. Sull'intonaco e oltre: diagnostica non distruttiva per il monitoraggio del patrimonio storico-monumentale misura in situ dell'effusività termica. 13a Conf. Nazionale sulle Prove non Distruttive Monitoraggio Diagnostica, Rome, Italy.

Ginzato, E., Cadelano, G., Bison, P., Peron, F., Maldague, X., 2010. High resolution and automatic survey of buildings by IR thermography. 10th Int. Conf. on Quantitative InfraRed Thermography, Québec, Canada.

Grinzato, E., 2012. State of art and perspective of Infrared Thermography applied to Building Science. In: Infrared Thermography. Recent Advances and future trends, Bentham Science publisher, pp. 200-229.

Ham, Y., Golparvar-Fard, M., 2013. An automated vision-based method for rapid 3D energy performance modeling of existing buildings using thermal and digital imagery, Advanced Engineering Informatics, 27, pp. 395-409.

Lagüela, S., Díaz-Vilariño, L., Martínez, J., Armesto, J., 2013. Automatic thermographic and RGB texture of as-built BIM for energy rehabilitation purposes. Automation in Construction, 31/2013, pp. 230-240.

Ministero dei Beni e delle attività Culturali e del Turismo, 2015. Linee di indirizzo per il miglioramento dell'efficienza energetica nel patrimonio culturale. Architettura, centri e nuclei storici ed urbani. Rome, Italy.

Pracchi, V., Rosina, E., 2013. Protecting Historical Buildings is not only a question of respecting their appearance. In: Proceedings of EWCHP 2013, 3rd European Workshop on Cultural Heritage Preservation, Bozen, Italy, pp. 211-218.

Previtali, M., Barazzetti, L., Redaelli, V., Rosina, E., Scaioni, M., 2013. A rigorous procedure for mapping thermal infrared images on 3D models of building façades, Journal of Applied Remote Sensing, 7(1), paper n. 073503.

Previtali, M., Barazzetti, L., Brumana, R., Cuca, B., Oreni, D., Roncoroni, F., Scaioni, M., 2014. Automatic façade modelling using point cloud data for energy efficient retrofitting, Applied Geomatics, 6(2), pp. 95-113.

Redaelli, V., Caglio, S., Gargano, M., Ludwig, N., Rosina, E., 2011. The surfaces of contemporary architecture: characterization of clinker by IRT. AITA Conf. 2011, L’Aquila, Italy.

Rosina, E., 2013. Assessment of historic buildings by infrared thermography. In: Thermography: Current status and advances in livestock animals and in veterinary medicine. Fondazione Iniziative Zooprofilattiche e Zootecniche Ed., Brescia, Italy, pp. 13-26.

Barazzetti L., Forlani G., Remondino, F., Roncella R., Scaioni M., 2011. "Experiences and achievements in automated image sequence orientation for close-range photogrammetric projects." In: Proc. Int. Conf. "Videometrics, Range Imaging, and Applications XI”, 23-26 May, Munich (Germany), Proc. of SPIE, Vol. 8085, paper No. 80850F, 13 pages, DOI: 10.1117/12.890116.

Scaioni, M., Rosina, E., Barazzetti, L., Previtali, M., Redaelli, V., 2012. "High-resolution texturing of building façades with thermal images." In: Proc. Int. Conf. "2012 Defense Security+Sensing,” 23-27 April, Baltimora (Maryland, USA), Proc. of SPIE, Vol. 8354, paper No. 83540I, 12 pages, DOI: 10.1117/12.920613.

Previtali, M., Erba, S., Rosina, E. Redaelli, V., Scaioni, M., Barazzetti, L., 2012. "Generation of a GIS-based environment for infrared thermography analysis of buildings.” In: Proc. Int. Conf. “2012 Infrared Remote Sensing and Instrumentation XX,” 12-16 Aug, San Diego (California, USA), Proc. of SPIE, Vol. 8611, paper No. 85110U, 12 pages, DOI: 10.1117/12.930050.

Stylianidis, E., Remondino, F., 2016.3D Recording, Documentation and Management of Cultural Heritage. Whittles Publishing, 388 pages.

Remondino, F., Spera, M.G., Nocerino, E., Menna, F., Nex, F., 2014: State of the art in high density image matching. Photogrammetric Record, Vol. 29(146), pp. 144-166.

Luhmann T, Robson S, Kyle S, Boehm J (2014) Close Range Photogrammetry: 3D Imaging Techniques $-2^{\text {nd }}$ Edition. Walter De Gruyter Inc., Germany, 684 pages.

Altuntas, C., Yildiz, F., Scaioni, M., 2016. Laser Scanning and Data Integration for Three-Dimensional Digital Recording of Complex Historical Structures: The Case of Mevlana Museum. ISPRS International Journal of Geo-Information, Vol. 5(18), 16 pages, DOI: 10.3390/ijgi5020018.

Saygi, G., Remondino, F., 2013. Management of Architectural Heritage Information in BIM and GIS: State-of-the-art and Future Perspectives. Int. Journal of Heritage in the Digital Era, Vol.2(4), pag. 695-714.

Snavely, N., Seitz, S.M. and Szeliski, R., 2008. Modeling the world from internet photo collections. International Journal of Computer Vision, 80(2): 189-210.

Westoby M.J., Brasington J., Glasser N.F., Hambrey M.J., Reynolds J.M. (2012) - 'Structure-from-Motion' photogrammetry: A low-cost, effective tool for geoscience applications. Geomorphology, 179: 300-314.

Hartmann, W., Havlena, M., Schindler, K., 2016. Recent Developments in Large-scale Tie-point Matching. ISPRS Journal of Photogrammetry and Remote Sensing, Vol. 115: 47-62.

Samet, H., 2006. Foundations of Multidimensional and Metric Data Structures. Morgan Kaufmann, 1024 pages.

Vollmer, M. and K.-P. Müllmann, 2010. Infrared Thermal Imaging. Fundamental Research and Applications. Wiley-VCH Verlag, Weinheim, Germany, 593 pages.

Gianinetto, M., Giussani, A., Roncoroni, F., Scaioni, M., 2005. Integration of Multi-Source Close-Range Data. Int. Arch. Photogramm. Remote Sens. Spatial Inf. Sci., Vol. XXXVI, Part 5/C34: 304-309.

Nocerino, E., Remondino, F., 2016. Uso consapevole di software speditivi per ricostruzioni 3D. Geomedia, Vol. 5/2016, pp. 40-42.

Luhmann, T., Fraser, C.S., Maas, H.-G., 2016. Sensor modelling and camera calibration for close-range photogrammetry. Journal of Photogrammetry and Remote Sensing, Vol. 115: 37-46. 